who had an equal "load" in terms of the product of the walking-response index and the recovery time. The ratio of the walking-response index to the recovery time showed essentially a u-shape against the walking distance, and one patients (case 7) who showed only an upswing was unable to discharge the hospital but others were able to discharge.
Our investigation suggests that a properly designed rehabilitation program for patients with severe respiratory failure requires careful judgement about when the patient is ready to begin training. Moreover, both the walking-response index and the recovery time are important determining factors of the distance each patient should be required to walk.

\title{
7-(1) (Supplementary) A Therapeutic Trial on its Acute Exacerbation
}

\author{
Yasunobu Fukushima, MD \\ The Second Department of Internal Medicine, Faculty of Medicine, \\ University of Toho, Tokyo
}

On a purpose of early efficient stabilization of gas exchange performance in the lung at acute exacerbation of chronic respiratory failure, we tried an additional therapeutic regimen with three drugs to the conventional procedures.

Oral administration of spironolacton $50-75$ $\mathrm{mg} /$ day and ubiquinone (Coenzyme $Q$ 10) 30 $\mathrm{mg} /$ day were taken for adjustment of ventilation perfusion interelations in lung functioning areas under their inotropic and antialdosteronic water balancing actions to the right ventricle and pulmonary interstices.

In comparison with 7 cases treated only with conventional medications, the 6 cases under this new regimen appeared to demonstrate more shift in $\mathrm{PaO}_{2}$ elevation $\left(13.05 \pm 6.24 *, 19.52 \pm 13.18^{*}\right.$, ns) and significant difference in $\mathrm{PaCO}_{2}$ reduction
$(0.06 \pm 6.05 *,-9.52 \pm 9.84 *, \mathrm{P}<0.05)$.

This group also indicated better access to the standard gas exchange ratio along the way of blood gas shift in therapeutic response.

Doxapram hydrochloride was used in a portable battery operated injection apparatus driven at the speed of $1-2 \mathrm{mg} / \mathrm{Kg} /$ hour.

This system was adopted on an idea to provide stable rhythmic performance of respiration, supporting if possible under better adaptation of more tidal volume and avoiding gas exchange insufficiency due to hypoventilation even during sleeping.

The results proved some sort and degree of improvement in alveolar ventilation except in a few cases of thoracic muscular weakness. ${ }^{*}$ Torr. 